\title{
X-RAY DIFFRACTION STUDY ON LATTICE VIBRATION OF FINE PARTICLES
}

\author{
Y. KASHIWASE, I. NISHIDA, Y. KAINUMA and K. KIMOTO \\ Physics Laboratory, Department of General Education, \\ Nagoya University, Chikusa-ku, Nagoya, Japan
}

\begin{abstract}
Résumé. - On a déterminé la température de Debye $\Theta_{\mathrm{M}}=156 \mathrm{~K}$, pour de petites particules d'argent d'environ $150 \AA$ A de diamètre à partir d'expériences de diffraction $\mathrm{X}$ relatives à la variation de l'intensité intégrée des anneaux de Debye-Scherrer en fonction de la température. Le fait que cette température de Debye soit bien inférieure à celle d'un cristal massif d'argent $\Theta_{M}=212 \mathrm{~K}$ permet de conclure à l'adoucissement des vibrations cristallines dans les petites particules d'argent.
\end{abstract}

\begin{abstract}
The Debye temperature $\Theta_{M}=156 \mathrm{~K}$ has been obtained for the fine particles of silver, whose size was about $150 \AA$ in diameter, from the X-ray diffraction experiment on the temperature dependence of the integrated intensity in the Debye-Scherrer rings. The fact that the Debye temperature obtained in the present experiment is much lower than that of the bulk silver crystal, i.e. $\Theta_{M}=212 \mathrm{~K}$, concludes the softening of lattice vibration occurring in the fine particles of silver.
\end{abstract}

1. Introduction. - Mean square amplitude of thermal vibration of atoms in the flat surface layers of a bulk crystal has been known to be from two to five times as large as that of atoms in the interior of the crystal $[1,2]$. The amplitude of atoms in the fine particles, whose size is less than about a hundred angström in diameter, may be expected to be remarkably large, since the number of atoms in the surface layers is comparable with that of atoms in the interior. Recently, particle size dependence of the Debye temperature has been found about the fine particles of silver [3], aluminium [3, 4, 5], indium [5] and lead [5]. These experimental results suggest the softening of lattice vibration in these small particles.

X-ray diffraction experiments have provided knowledges about the Debye-Waller factors and the X-ray Debye temperature $\Theta_{M}$ for the bulk crystals of many substances. In the case of powder specimen experiment, specimens were prepared by, for example, electrolysis, chemical precipitation and machining the polycrystalline material and the size of the specimen particles was rather large. It was of the order of a few $\mu \mathrm{m}$ in most cases.

$\mathrm{X}$-ray studies on thermal motion of atoms in the fine particles whose size was less than several hundred angströms were first carried out by using the silver [6] and gold [7] particles prepared by gas evaporation technique [8, 9]. By using electron diffraction the present authors already reported the anomalously large mean square displacement of atoms in the fine particles of silver [6] and $\delta$ chromium [6], whose sizes were about $20 \sim 50 \AA$ in diameter, at room temperature. The results were obtained from the measurements of integrated intensities of Bragg reflections at different scatter- ing angles in the Debye-Scherrer rings of electron diffraction patterns. Since temperature dependence of the intensity was not measured from experimental difficulty in electron diffraction, there remained a possibility that a part of the observed mean square displacement might be due to the static distortion of the particles.

The aim of the present paper is to report the result of X-ray measurement of temperature dependence of the intensities at given reflections from the fine particles of silver whose size was about $150 \AA$ in diameter.

2. Theory. - In the case of the kinematical theory of X-ray diffraction from a powder sample of crystallites with a simple structure consisting of one kind of atom of mass $m$ in a unit cell under harmonic thermal vibration, we have the equation

$$
\frac{1}{2} \ln \left(I_{\mathrm{c}} / I\right)=B(\sin \theta / \lambda)^{2},
$$

where $I$ is the observed integrated intensity per unit length along the Debye-Scherrer ring at the Bragg angle $\theta, I_{c}$ the integrated intensity calculated from the powder specimen of small crystals consisting of static atoms, and $\lambda$ the wavelength of radiation used. The Debye parameter $B$ is related to the mean square amplitude $\left\langle\boldsymbol{u}^{2}\right\rangle$ of thermal vibration of atoms by the equation

$$
B=8 \pi^{2}\left\langle u^{2}\right\rangle / 3,
$$

on the assumption of isotropic thermal vibration. From the slope of curve about $\frac{1}{2} \ln \left(I_{c} / I\right)$ versus $(\sin \theta / \lambda)^{2}$, the values of $B$-factor and $\left\langle u^{2}\right\rangle$ can be determined. The $B$-factor is the sum of zero point 
energy parameter $B_{0}$ and the thermal Debye parameter $B_{\mathrm{T}}$ which are related to the Debye temperature $\Theta_{M}$ as

$$
\begin{aligned}
& B_{0}=\left(6 h^{2} / m k_{\mathrm{B}} \Theta_{\mathrm{M}}\right) / 4 \\
& B_{\mathrm{T}}=\left(6 h^{2} / m k_{\mathrm{B}} \Theta_{\mathrm{M}}\right) \phi(x) / x
\end{aligned}
$$

with

$$
x=\Theta_{M} / T
$$

and

$$
\phi(x)=\frac{1}{2} \int_{0}^{x} \xi /\left(e^{\xi}-1\right) d \xi,
$$

where $h$ and $k_{\mathrm{B}}$ are the Planck constant and the Boltzmann constant, respectively. On the assumption of temperature independent Debye temperature, or harmonic thermal vibration, we have the equation

$$
\begin{aligned}
& \ln \left(I(T) / I\left(T_{0}\right)\right) /\left[2(\sin \theta / \lambda)^{2}\right]= \\
& =\left(6 h^{2} / m k_{\mathrm{B}} \Theta_{\mathrm{M}}\right)\left(\phi\left(x_{0}\right) / x_{0}-\phi(x) / x\right),
\end{aligned}
$$

where $I(T)$ is the integrated intensity of a DebyeScherrer ring from the specimen at temperature $T$. $T_{0}$ and $x_{0}$ represent reference temperature of the specimen and $\Theta_{\mathrm{M}} / T_{0}$, respectively. The Debye temperature can be determined also from the slope of the curve given by the equation.

$$
\begin{aligned}
\ln \left(I(T) / I\left(T_{\mathrm{o}}\right)\right) /[ & \left.2(\sin \theta / \lambda)^{2}\right]= \\
= & \left(6 h^{2} / m k_{\mathrm{B}}\right)\left(T_{\mathrm{o}}-T\right) / \Theta_{\mathrm{M}}^{2}
\end{aligned}
$$

in the high temperature limited approximation $\phi(x)=1$ which can be used at temperature $T \gtrsim \Theta_{M}$.

3. Experimental Procedure. - The silver particles whose size was about $150 \AA$ in diameter were prepared by gas evaporation technique. Figure 1 shows the specimen particles of silver. The most

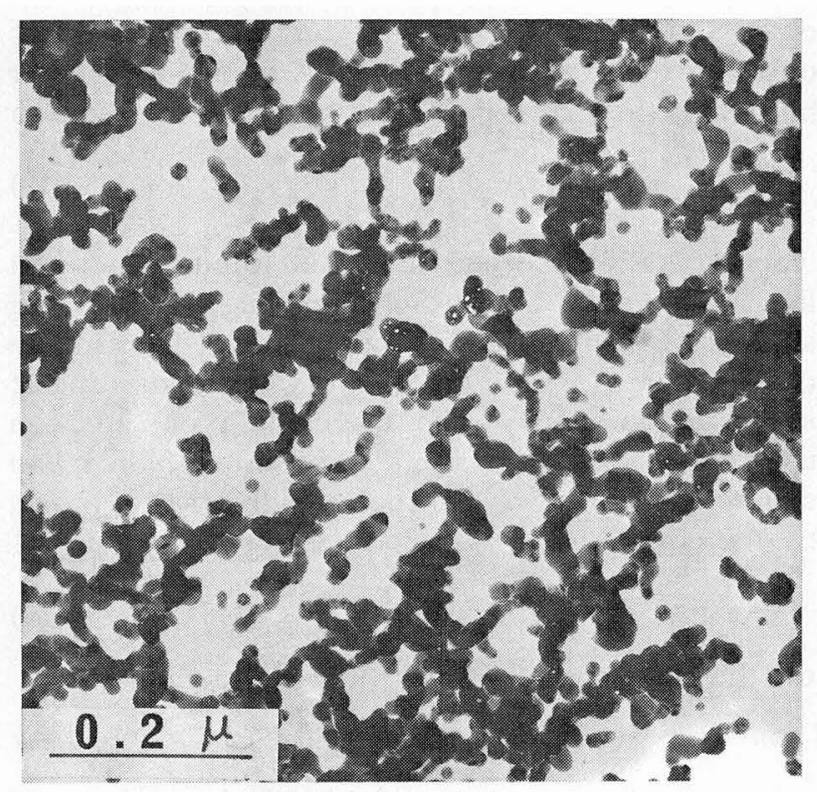

FIG. 1. - Electron micrograph of the specimen fine particles of silver produced in helium at gas pressure 8 torr. particles look round and linked together. These particles do not show well-defined crystal habit. The contrast of the particles varies according to the location in the linkage. Some of the isolated particles are in hexagonal profile. They show a characteristic contrast identified as multiplytwinned particles [10]. The size distribution of the particles in figure 2 shows that the mean size of the

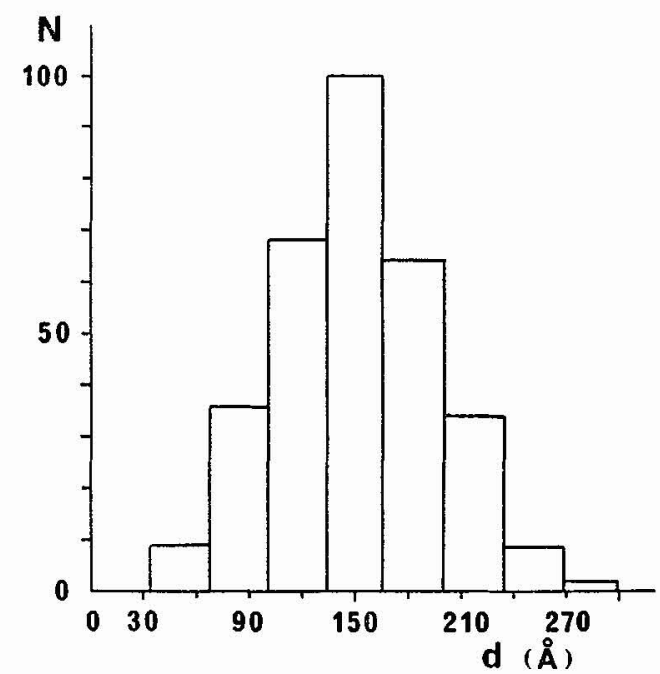

FrG. 2. - Size distribution of the specimen particles. Number of particles $N$ versus particle size $d$ in diameter. Peak value is normalized to 100 .

specimen particles used is about $150 \AA$. The particle size measured from electron microscope image was consistent with that measured from line breadth of the Debye-Scherrer rings by using Scherrer's formula. The sample was filled in a beryllium tube of $10 \mathrm{~mm}$ length and of inside diameter $0.27 \mathrm{~mm}$ and outside diameter $0.35 \mathrm{~mm}$. The packing density was about $20 \%$. The tube was mounted on the specimen holder in the vacuum chamber of cryostat. Molybdenum $\mathbf{K}_{\alpha}$ X-rays through yttrium and zirconium balanced filter were used. The intensity distributions of the DebyeScherrer rings from the specimen at given temperature were measured by means of scintillation counter, using monitoring system and step scanning technique. The intensity was very weak. The peak intensity observed in 220 reflection was about 5 . counts per second. The line breadth were so broad that separation of intensity distributions due to $\alpha_{1}$ and $\alpha_{2}$ components of the radiation used was not observed at 422 reflection. The scattering and the absorption by the beryllium tube were negligibly small because of much lower scattering power than that of the sample used and low absorption coefficient. The problem of the absorption correction for $I_{\mathrm{c}}$ was not important, since the absorption of Molybdenum $\mathrm{K}_{\alpha}$ by the sample was so low that the absorption correction was almost constant in the range of scattering angle observed. Temperature of the specimen measured with a chromel- 
alumel thermocouple agreed with that measured from the shift of the peak position of the Bragg reflection on the assumption that the expansion coefficient of the specimen particles is the same as that of the bulk crystals.

4. Result. - The typical intensity distribution and the temperature variation of 220 reflection are shown in figure 3 . Open circles and solid circles in figure 3 represent respectively the intensities observed at specimen temperature $294 \mathrm{~K}$ and $104 \mathrm{~K}$ at each scattering angle.

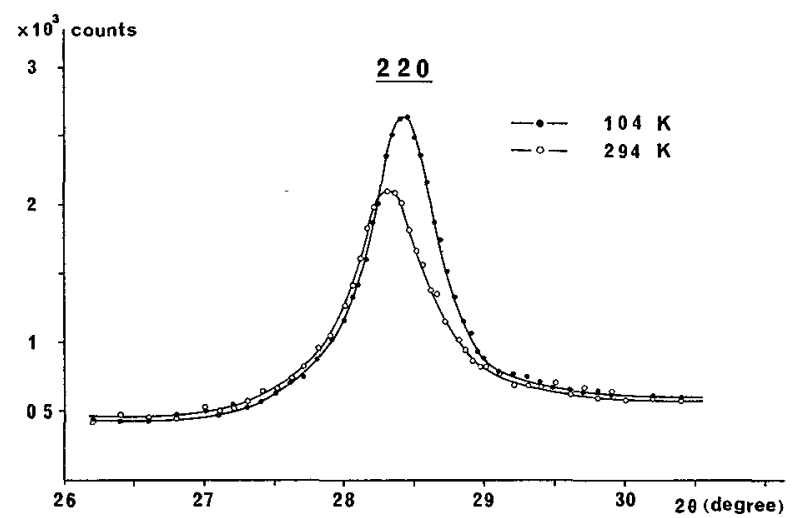

FIG. 3. - Intensity distribution and the temperature variation of 220 reflection.

The experimental result of the temperature variation of the integrated intensity of 220 reflection from a smoke sample is shown in figure 4 . The experiments of the temperature variation were carried out repeatedly several times by using the same sample. The plots obtained by a single experimental run are shown by the same marks. Error bar represents the accidental error of the observed intensity. The value of the Debye temperature $\Theta_{\mathrm{M}}=156 \pm 3 \mathrm{~K}$ is obtained from eq. (8) and from the slope of the solid curve $(a)$ which satisfies the least square fitting of the experimental plots. The dashed curve $(b)$ in the figure represents the intensity variation calculated from eq. (8) by using

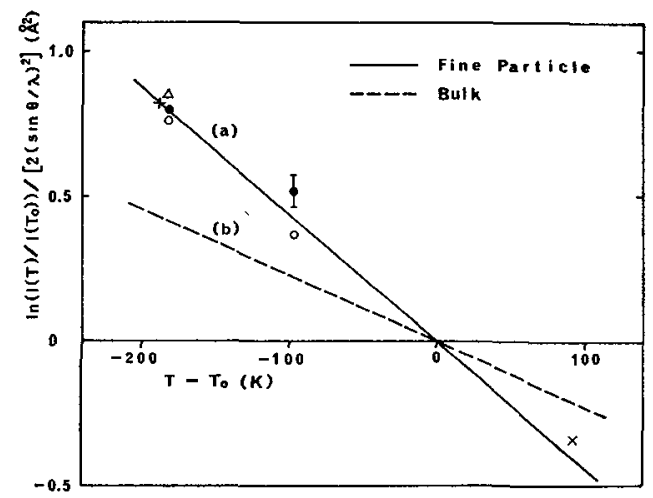

FIG. 4. - Temperature variation of the integrated intensity of 220 reflection from $(a)$ the fine particles and $(b)$ the bulk of silver. $T_{0}$ is room temperature. the Debye temperature of bulk silver crystal $\Theta_{\mathrm{M}}=212 \mathrm{~K}$.

The open circles and the solid circles shown in figure 5 represent the experimental results obtained

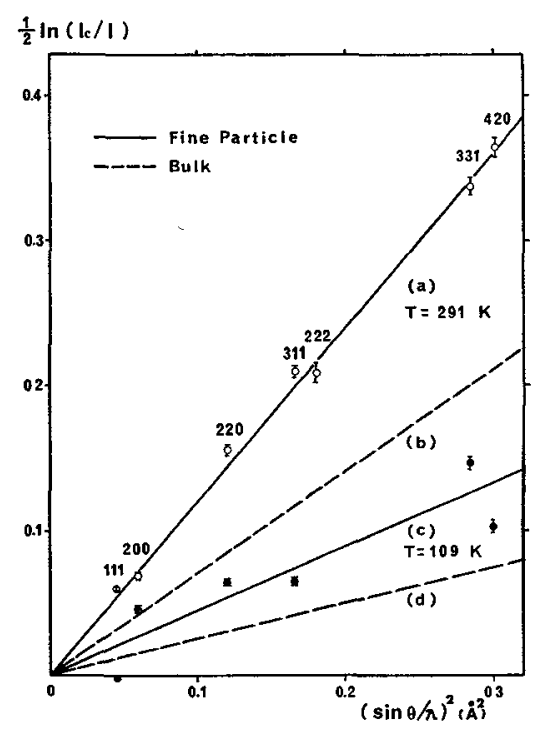

FIG. 5. - The scattering angle dependence of the integrated intensity of Bragg reflections from silver particles. The marks and $\$$ express the experimental plots at $291 \mathrm{~K}$ and $109 \mathrm{~K}$, respectively.

from the reflections $111,200,220,311,222,331$ and 420 of the same samples described above at $291 \mathrm{~K}$ and $109 \mathrm{~K}$, respectively. Temperature variation of the value of the Debye parameter $B$ can be seen from the temperature variation of the slope of curves in the figure. The curves $(a)$ and $(c)$ in the figure were obtained by the least square fitting method. The values of the $B$ factors for the curves $(a)$ and $(c)$ are $1.18 \AA^{2}$ and $0.43 \AA^{2}$, respectively. The value of the Debye temperature $\Theta_{M}$ obtained from the temperature variation of the slope of the curves $(a)$ and (c) in the high temperature limit approximation is $161 \pm 7 \mathrm{~K}$. Agreement of this Debye temperature with that obtained by the method described above is satisfactory. The dashed curves $(b)$ and $(d)$ in figure 5 are drawn by calculation using the Debye temperature $212 \mathrm{~K}$ of bulk crystal in eq. (1) at $291 \mathrm{~K}$ and $109 \mathrm{~K}$, respectively.

5. Conclusion and Discussion. - The Debye temperature $\Theta_{M}=156 \mathrm{~K}$ obtained in the present experiment is much lower than the Debye temperature $\Theta_{M}=212 \mathrm{~K}$ of the bulk silver crystal. The corresponding mean square amplitude of thermal vibration of atoms in the silver fine particles observed is about two times as large as those of bulk crystal. This fact concludes that the softening of lattice vibration is observed in the fine particles of silver of size $150 \AA$ in diameter. The value of the Debye temperature and the mean square amplitude 
observed for the silver fine particles as a whole are close to those of the atoms in the flat surface layer of the bulk crystal which are measured by the experiment of low energy electron diffraction. This means that the surface effect on the lattice vibration observed in the bulk crystal penetrates into the interior of the fine particles and causes the softening of the lattice vibration.

Remarkable effect of surface on lattice vibration of a fine particles suggest melting point much lower than that of bulk crystal. It is well-known $[11,12$, 13] that metallic fine particles have melting points much lower than those of the corresponding bulk metals. The present lowering of the Debye temperature is consistent with the fact described above. Assumptions of harmonic thermal vibration and temperature independent Debye temperature can be justified for the fine particles of silver studied in the present experiment, since the deviation of the experimental plots from the theoretical curves obtained from assumption of harmonic vibration is small as seen in figures 4 and 5 . It, however, should be noticed that the deviation of experimental plots from the least square fitting curve is rather large for the specimen at temperature $109 \mathrm{~K}$ in figure 5. This might be due to such static distortions of the lattice as those included in the multiply-twinned particles. The study on the effect of the static distortion in the integrated intensity is left to the future.

The correction of the integrated intensity for a contribution from the temperature diffuse scattering is neglected in the present experiment. The correction will lower the value of the Debye temperature. Decrease of the Debye temperature due to the correction, however, is expected to be small, since the indexes of reflection planes used are low. The lowering of the Debye temperature from the experiment about temperature dependence of electrical conductivity in films of fine particles of silver [3] is in accord with the present result.

\section{References}

[1] Morabito, J. M., Steiger, R. F. and Somorjai, G. A., Phys. Rev. 179 (1969) 638.

[2] Wallis, R. F., Progress in Surface Science ed. Davison, S. G. (Pergamon Press, Oxford, 1974) Vol. 4, p. 233

[3] Fujita, T., Oshima, K. and Kuroishi, T., J. Phys. Soc. Japan 40 (1976) 90.

[4] Matsuo, S., Sugryama, H. and Noguchi, S., J. Low Tem. Phys. 15 (1974) 481.

[5] Matsuo, S., Miyata, H. and Noguchi, S., Jpn J. Appl. Phys. 13 (1974) 351.

[6] Kashiwase, Y., Nishida, I., KaINUMA, Y. and Krmoto, K., J. Phys. Soc. Japan 38 (1975) 899.
[7] Harada, J., YaO, S. and IChimiYa, A., Acta Crystallogr. A 31 (1975) 204.

[8] Kimoto, K., Kamiya, Y., Nonoyama, M. and Uyeda, R., Japan J. Appl. phys. 2 (1963) 702.

[9] Kimoto, K. and NishiDA, I., Japan J. Appl. Phys. 6 (1969) 104.

[10] Ino, S., J. Phys. Soc. Japan 21 (1966) 346.

[11] TAKaGI, M., J. Phys. Soc. Japan 9 (1954) 359.

[12] Blackman, M. and CURSon, A. E., Structure and Properties of Thin Films ed. Neugebauer, Newkirk and Vermilyea (John Wiley \& Sons, Inc. New York), 1959, p. 217.

[13] Berman, R. P. and Curzon, A. E., Can. J. Phys. 52 (1974) 923. 